\title{
Diagnóstico de las percepciones de los factores de riesgo psicosociales en el trabajo del personal de una industria manufacturera
}

\author{
Diagnosis of the perceptions of psychosocial risk \\ factors in the work of the personnel \\ of a manufacturing industry
}

Estibalis Anaís LUNA-CHÁVEZ1 ID 0000-0003-4296-6295

Ana ANAYA-VELASCO ${ }^{1}$ (D) 0000-0003-1137-9645

Ezequiel RAMÍREZ-LIRA ${ }^{1}$ (iD) 0000-0002-6768-8941

\section{Resumen}

Los factores psicosociales de riesgo están en el entorno del trabajador y su percepción depende de diversas causas. Este estudio, no experimental y transversal fue realizado en una industria manufacturera del sur de Jalisco, México, el objetivo fue evaluar la percepción del personal sobre los factores psicosociales, mediante la Batería Condiciones de Trabajo de Carácter Psicosocial. El personal mostró una percepción de muy bueno y bueno para Factores Individuales con 84.9\%, que los hace probablemente protectores de Desgaste Psíquico, mientras que para Contenido de Trabajo fue de $55.2 \%$ y para Contexto de Trabajo apenas de $40.2 \%$, dando como diferencia $44.8 \%$ y $59.8 \%$ respectivamente, una percepción de normal, regular y nocivo, convirtiéndolos en probables factores de riesgo psicosocial para Desgaste Psíquico, lo que mostró la necesidad de acciones de mejora.

Palabras clave: Factores psicosociales; Organización; Salud ocupacional.

\begin{abstract}
Psychosocial risk factors are present in the worker's environment and his perception about them depends on a variety of causes. This study, a non-experimental and cross-sectional one, was carried out in a manufacturing industry in southern
\end{abstract}

1 Universidad de Guadalajara, Centro Universitario del Sur, Posgrado en Psicología con Orientación en Calidad de Vida y Salud. Av. Enrique Arreola Silva, 833, Colonia Centro, 49000, Guzmán, Jalisco, México. Correspondencia para/Correspondence to: E. RAMÍREZ-LIRA. E-mail: <ezequielr79@gmail.com>.

Artículo basado en la tesis de E.A. LUNA-CHÁVEZ, titulada "Propuesta de intervención en una industria del sur de Jalisco, con el modelo de salud y seguridad en el trabajo con gestión integral para la sustentabilidad". Universidad de Guadalajara, 2018.

$\boldsymbol{\nabla} \mathbf{v} \boldsymbol{\nabla}$

Como citar este artículo/How to cite this article

Luna-Chávez, E. A., Anaya-Velasco, A., \& Ramírez-Lira, E. (2019). Diagnóstico de las percepciones de los factores de riesgo psicosociales en el trabajo que tiene el personal de una industria manufacturera. Estudos de Psicologia (Campinas), 36, e1800148. http://dx.doi. org/10.1590/1982-0275201936e180148
} 
Jalisco, Mexico, whose objective was to evaluate the perception of personnel on psychosocial factors, through the Working Conditions of Psychosocial Character Battery. Regarding the company staff, $84.9 \%$ of the workers rated their perception of Individual Factors as good or very good, which makes them likely immune to Psychological Exhaustion, while for the Work Content variable, 55.2\% of them rated their perception as good or very good, and for Work Context just $40.2 \%$, resulting in a difference of $44.8 \%$ and $59.8 \%$ respectively, a perception of normal, regular and harmful conditions, which turned them into a vulnerable psychosocial risk factor group for Psychological Exhaustion, which highlighted the need for improvement actions.

Keywords: Psychosocial factors; Organization; Occupational health.

En el contexto organizacional, no se está exento de accidentes, lesiones y enfermedades debido a los factores de riesgo presentes. La Organización Internacional del Trabajo (2013) señaló que, aunque algunos de los riesgos tradicionales han disminuido gracias a la seguridad, a los adelantos técnicos y a la normatividad existente, éstos siguen afectando la salud de los trabajadores ya que se registra un aumento de nuevas enfermedades profesionales sin que se apliquen medidas de prevención, protección y control adecuadas. Entre los riesgos emergentes se incluyen los factores psicosociales, que son producto de la inadecuada organización del trabajo, debido a la cultura, tanto del individuo como de la organización (Labarthe \& Fillat, 2017).

En el marco del Día Mundial de la Seguridad y Salud en el Trabajo 2016, la Oficina Internacional del Trabajo (OIT) señaló que la atención de estos factores es un problema mundial que preocupa a todos los países y a todos los trabajadores. En este ámbito, el centro de trabajo es una fuente importante de riegos psicosociales y al mismo tiempo un lugar idóneo para tratarlos y también proteger la salud y el bienestar de los trabajadores, su desempeño y los resultados de la organización. Los factores psicosociales que existen en el mundo y, en particular en México, exigen un compromiso gubernamental para fortalecer la seguridad y la salud en el trabajo, de modo que éste sea digno y decente. Para eso se deben implementar políticas, líneas estratégicas de acción y proyectos con un enfoque preventivo, para que prevalezcan centros de trabajo con condiciones seguras y saludables (Secretaría de Trabajo y Previsión Social, 2015).

En ese ámbito, la OIT en conjunto con la Organización Mundial de la Salud definieron en 1984 (Comité Mixto OIT/OMS de Medicina del Trabajo, 1984) los factores de riesgo psicosocial como aquellas condiciones presentes en una situación de trabajo, relacionadas a la organización, al contenido y a la realización de la tarea, condiciones estas susceptibles de afectar tanto el bienestar y la salud física, psíquica o social de los trabajadores como el desarrollo de la labor, causando estrés e incluso burnout (García, Puebla, Herrera, \& Daudén, 2016). Esta idea ha ido evolucionando hasta ser respaldada por la legislación tanto internacional como nacional, sin embargo, el concepto de factores psicosociales alude a interacciones entre el trabajo, el medio ambiente, la satisfacción en el trabajo y las condiciones de organización, así como a las capacidades del trabajador, sus necesidades, su cultura y su situación personal fuera del trabajo considerando sus experiencias y percepciones.

Los factores psicosociales pueden ser factores de riesgo para la salud con origen en la organización del trabajo generando respuestas de tipo fisiológico, emocional, cognitivo y conductual que son conocidas popularmente como estrés y que pueden ser precursoras de enfermedad en ciertas circunstancias de intensidad, frecuencia y duración (Instituto Sindical de Trabajo, Ambiente y Salud, 2015; Marín \& Piñeros, 2018). Estos factores resultan de deficiencias en el diseño, la organización y la gestión del trabajo. Algunos ejemplos de condiciones de trabajo que entrañan riesgos psicosociales son: carga de trabajo excesiva, exigencias contradictorias y falta de claridad de las funciones del puesto, falta de participación en la toma de decisiones que afectan al trabajador y falta de influencia en el modo en que se lleva a cabo el trabajo, gestión deficiente de los cambios organizativos, inseguridad en el empleo, comunicación ineficaz, falta de apoyo por parte de la dirección o los compañeros, acoso psicológico y sexual, violencia ejercida por terceros, insatisfacción y baja motivación (Agencia Europea para la Salud y la Seguridad en el Trabajo, 2016; Hernán, 2018; Luceño,

2 Talavera, Martín, \& Escorial, 2017). Por ello, proponer acciones dirigidas al control de los mismos, centrados en 
el talento humano y desde una perspectiva multidisciplinaria, creará un proceso participativo y de pertenencia (Katekamp et al., 2014; Moreno \& Báez, 2010).

Es indispensable no confundir las exigencias propias del trabajo con los factores psicosociales como una carga de trabajo excesiva o de riesgo con situaciones que, aunque estimulantes y a veces desafiantes, ofrecen un entorno de trabajo en el cual se apoya al trabajador, que recibe la formación adecuada y está motivado para desempeñar su trabajo de la mejor manera posible (Agencia Europea para la Salud y la Seguridad en el Trabajo, 2016).

Se trata entonces de formar entornos psicosocialmente favorables con el objetivo de fomentar el buen rendimiento y el desarrollo personal, así como el bienestar mental y físico del trabajador. De lo contrario, los efectos negativos se traducen en un mal rendimiento global de la empresa, aumento del ausentismo, presentismo (cuando el trabajador acude a su empleo estando enfermo, y por ello es incapaz de rendir con eficacia) y mayores índices de accidentes y lesiones. Las bajas tienden a ser más prolongadas que las derivadas de otras causas, y el estrés relacionado con el trabajo puede contribuir a un aumento de los índices de jubilación anticipada (Agencia Europea para la Salud y la Seguridad en el Trabajo, 2016; Cacivio, 2017; Vera, Carrasco, Da Costa, \& Paez, 2015).

Hacer caso omiso o prestar poca atención a los factores de riesgo psicosociales dentro de una organización pueden generar costos e impactos negativos tanto para las empresas, ya sea directa o indirectamente, como para los trabajadores, sus familias e incluso para la sociedad, debido a que se ve afectado el ingreso económico y también se genera costo para las instituciones de seguridad social (Mendoza, 2013).

En México, se reglamentó la necesidad de identificar, evaluar y controlar los factores de riesgo psicosocial publicándola en el Diario Oficial de la Federación en 2014 (Secretaría del Trabajo y Previsión Social, 2014). Posteriormente el 23 de octubre de 2018, se publicó en el Diario Oficial de la Federación, la Norma Oficial Mexicana NOM-035-STPS-2018, la cual ofrece una serie de estrategias para la identificación, análisis y prevención de los factores de riesgo psicosocial, la violencia laboral, y la promoción del entorno organizacional favorable; acciones que toda organización debe acatar (Secretaría del Trabajo y Previsión Social, 2018). De acuerdo con lo anterior, para tener un mejor control en la prevención de los factores psicosociales, las políticas deben esforzarse por involucrar a las organizaciones en las buenas prácticas, utilizando para su diagnóstico instrumentos precisos, contextualizados y, establecer estándares para la óptima salud ocupacional, por medio de legislaciones o acciones de intervención (Potter, O’Keeffe, Leka, Webber, \& Dollard, 2019; Unda et al., 2016; Vazquez, Pianezolla, \& Hutz, 2018; Weissbrodt, Arial, Graf, Iff, \& Giauque, 2018).

Las condiciones de trabajo y su relación con los factores psicosociales de riesgo, a las que el personal de una organización está sometido, impactan negativamente tanto en su salud física, mental y emocional, como en el entorno laboral (Anaya, 2016; Anaya, Saldaña, \& Ramírez, 2017; Carrión, Gutiérrez, \& López, 2014b; Saldaña, Ramírez, Anaya, \& Baeza, 2014), por lo que el Desgaste Psíquico, se define como los factores que contemplan el esfuerzo de atención y concentración necesarios para recibir las informaciones del proceso y elaborar las respuestas adecuadas y que por ser constante durante la jornada, debe ser sostenido. En conjunto, la fatiga percibida, los síntomas subjetivos y alteraciones de la salud son consecuencias que se desprenden de una sobrecarga de las exigencias de la tarea, también el número de informaciones que se precisan para realizar la tarea y el nivel de complejidad de las mismas; y finalmente, la percepción de la dificultad que para el trabajador tiene su trabajo (Instituto Nacional de Seguridad e Higiene en el Trabajo, 1995), es por ello que a partir de un buen diagnóstico, con instrumentos y técnicas precisas de acuerdo con el contexto de trabajo, es posible establecer líneas de control para aminorar la presencia de los factores de riesgo psicosocial percibidos y así mejorar el bienestar y calidad de vida del trabajador (Mathisen, Bronnick, Arntzen, \& Vestly, 2016, Potter et al., 2019; Thomascheck, Lütke, Melzer, Debitz, \& Buruck, 2017; Vestly, Leka, \& Zwetsloot, 2017). 
El presente estudio se realizó con el propósito de evaluar la percepción del personal de una industria manufacturera sobre los factores psicosociales en relación con el desgaste psíquico y proponer medidas dirigidas al control de los factores psicosociales de riesgo encontrados. La empresa del ramo papelero del sur de Jalisco, México, la cual es parte de un corporativo a nivel nacional con importante reconocimiento. Se rige bajo la Ley Federal del Trabajo, la Normativa Oficial Mexicana y las políticas de gestión integral que comparten en todo el corporativo. El personal está dividido en 12 áreas diferentes para llevar a cabo sus actividades diarias. La calidad y productividad de sus procesos organizacionales se basan en la eficiencia de sus servicios y la óptima satisfacción de sus clientes.

\section{Método}

El estudio fue cuantitativo, no experimental, transversal, de tipo descriptivo de los factores de riesgo psicosocial y correlacional de éstos con el desgaste psíquico (Kerlinger \& Lee, 2002).

\section{Participantes}

La selección de la organización fue por conveniencia (Coolican, 2005). Del universo de 246 trabajadores, 199 autorizaron su participación por medio de un consentimiento informado; 39.2\% fueron hombres y $60.8 \%$ mujeres. La escolaridad reportada por los participantes fue: $1.5 \%$ del personal sin formación escolar, $17.5 \%$ estudiaron hasta el nivel primario, $36.7 \%$ hasta el nivel secundario, $24.6 \%$ con nivel de bachillerato, $8.5 \%$ con formación profesional, $10.1 \%$ con nivel licenciatura y $1.0 \%$ con posgrado.

\section{Instrumentos}

Batería para el estudio de las Condiciones de Trabajo de Carácter Psicosocial (CTCPS-MAC), desarrollada y validada por Carrión (2014a) en población española y posteriormente validada en población latinoamericana (Colombia, Ecuador, México, Perú y Venezuela) por un grupo de expertos. Fue diseñada para evaluar la percepción que tiene la población trabajadora sobre los factores psicosociales laborales en su propio entorno de trabajo, así como factores individuales, síntomas subjetivos y alteraciones de la salud. Posee una consistencia interna con el alfa de Cronbach de 0.92. La batería consta de 75 ítems que agrupan cuatro dimensiones como factores psicosociales globales y 14 factores psicosociales específicos integrados en las cuatro dimensiones (Tabla 1).

La opción de respuesta es de tipo Likert que va desde estoy completamente en desacuerdo hasta estoy completamente de acuerdo y su calificación se encuentra en los niveles muy bueno, bueno, normal, regular y nocivo.

\section{Procedimiento}

Se estableció contacto con la empresa participante y después se solicitó la autorización de su parte para lo requerido por este estudio. Se administró la batería CTCPS-MAC considerando las condiciones óptimas que establece el manual de aplicación. Se les explicó a las personas que decidieron participar en qué consistía la batería y se procedió a la obtención de las respuestas a la misma. Posteriormente se recolectaron y analizaron los datos por medio del Statistical Package for the Social Sciences 21.0 para obtener los niveles

4 de riesgo percibidos por el trabajador por medio de la estadística descriptiva y correlacional. Con base en 
Tabla 1

Componentes de la Batería Condiciones de Trabajo de Carácter Psicosocial

\begin{tabular}{ll}
\hline Dimensión & Factores psicosociales \\
\hline D1-F1. Cultura de la Organización y Gestión \\
D1-F2. Papel o Rol en la Organización \\
D1-F3. Interrelación Trabajo con Problemas Familiares o Sociales \\
D1-F4. Relaciones Interpersonales \\
D2-F1. Carga y Ritmo de Trabajo \\
D2-F2. Ambientes Laborales \\
D2-F3. Equipos y Agentes Físicos \\
D2-F4. Concepción de las Tareas del Puesto de Trabajo \\
D3-F1. Amortiguadores del Riesgo Psíquico \\
D3-F2. Características de la Empresa \\
D3-F3. Características Personales \\
D4-F1. Síntomas subjetivos y alteraciones de la salud - Estados psicológicos - Respuesta Cognitiva \\
D4-F2. Síntomas subjetivos y alteraciones de la salud - Estados psicológicos - Respuesta Conductual \\
D4-F3. Síntomas subjetivos y alteraciones de la salud - Estados psicológicos - Respuesta Fisiológica
\end{tabular}

Nota: Elaboración propia a partir de la batería Condiciones de Trabajo de Carácter Psicosocial (Carrión et al., 2014b).

ello, se propusieron líneas de acción para mejorar las condiciones de trabajo psicosociales percibidas como probablemente dañinas con calificación de normal, regular y nocivo y por tanto probables factores de riesgo psicosocial. También se consideraron otras medidas complementarias para mantener las condiciones psicosociales probablemente protectoras percibidas con calificación de muy bueno y bueno.

\section{Resultados}

Los resultados obtenidos en porcentaje por dimensión psicosocial de la batería CTCPS-MAC se muestran en la Tabla 2, donde se observa el predominio de la percepción de los trabajadores en nivel de muy bueno y bueno para la dimensión Factores Individuales, mientras que para la de Contenido de Trabajo fue de poco más de la mitad de los participantes y para Contexto de Trabajo apenas de $40.2 \%$, dando como diferencia, una alta percepción de normal, regular y nocivo, lo cual exige establecer medidas para mejorar esas calificaciones. No obstante, lo observado en las dimensiones referidas como variables independientes, el Desgaste Psíquico, considerado como variable dependiente, resultó con una alta incidencia de respuesta en muy bueno y bueno similar a la de los Factores Individuales.

Tabla 2

Frecuencia y porcentaje de las dimensiones de la batería Condiciones de Trabajo de Carácter Psicosocial

\begin{tabular}{|c|c|c|c|c|c|c|c|c|c|c|}
\hline \multirow{2}{*}{ Dimensión } & \multicolumn{2}{|c|}{ Muy bueno } & \multicolumn{2}{|c|}{ Bueno } & \multicolumn{2}{|c|}{ Normal } & \multicolumn{2}{|c|}{ Regular } & \multicolumn{2}{|c|}{ Nocivo } \\
\hline & fr & $\%$ & $f r$ & $\%$ & $f r$ & $\%$ & fr & $\%$ & fr & $\%$ \\
\hline Contexto de Trabajo & 8 & 4.0 & 72 & 36.2 & 3 & 1.5 & 108 & 54.3 & 8 & 4.0 \\
\hline Contenido de Trabajo & 11 & 5.5 & 97 & 49.7 & 12 & 6.0 & 78 & 39.2 & 1 & 0.5 \\
\hline Factores Individuales & 34 & 17.1 & 135 & 67.8 & 5 & 2.5 & 24 & 12.1 & 1 & 0.5 \\
\hline Desgaste Psíquico & 50 & 25.1 & 117 & 58.8 & 2 & 1.0 & 29 & 14.6 & 1 & 0.5 \\
\hline
\end{tabular}

Nota: Elaboración propia a partir de la batería Condiciones de Trabajo de Carácter Psicosocial (Carrión et al., 2014b). 
Posteriormente se procedió a conocer el porcentaje y frecuencia por factor psicosocial específico de la Batería CTCPS-MAC (Tabla 3). De acuerdo con los resultados mostrados en esa tabla, se indica que entre la mitad y las tres cuartas partes del personal evaluado percibió como "muy bueno" y "bueno" 6 de los 11 factores psicosociales como variables independientes; equipos y agentes físicos, y carga y ritmo de trabajo de la dimensión contenido de trabajo, seguidos de relaciones interpersonales, interrelación del trabajo con problemas familiares y sociales, y papel o rol en la organización de la dimensión contexto de trabajo; los más presentes fueron los amortiguadores del riesgo psíquico, las características personales y características de la organización siendo los tres de la dimensión de factores individuales. Mientras que, en los otros tres factores ambientes laborales, concepción de las tareas del puesto de trabajo y cultura de la organización y la gestión resultó lo inverso, sólo una tercera parte del personal los percibió en nivel "muy bueno" y "bueno". Por diferencia con esta incidencia, la respuesta en normal, regular y nocivo es alta con la probabilidad de causar Desgaste Psíquico, por lo cual se requieren medidas de acción que mejoren estas condiciones.

No obstante, los factores respuesta cognitiva-emocional, respuesta conductual y respuesta fisiológica de la dimensión de desgaste psíquico, mostraron entre $67.8 \%$ y $80.9 \%$ de trabajadores con percepción en nivel "muy bueno" y "bueno"; por diferencia, el porcentaje de personal que tiene percepción en nivel normal, regular y nocivo es importante, confirmando la exigencia de medidas de mejora.

Se llevó a cabo una correlación de Pearson para ver la asociación de las dimensiones psicosociales con el desgaste psíquico. Los resultados indicaron una correlación media, positiva y significativa (Coolican, 2005) de las dimensiones contexto de trabajo, contenido de trabajo y factores individuales con la dimensión de desgaste psíquico por lo que es posible inferir que dichas condiciones psicosociales de riesgo en el trabajo se asocian a las respuestas tanto cognitiva-emocional, conductual como físicas (Tabla 4).

Lo anterior muestra la necesidad de actuar principalmente en contexto de trabajo y contenido de trabajo, con medidas dirigidas a disminuir e incluso, mejorar las condiciones más desfavorables percibidas por el personal de esta industria por lo que se procedió a realizar un listado con propuestas de acciones para el cumplimiento de dicho objetivo.

Tabla 3

Frecuencia y porcentaje de los factores de la Batería Condiciones de Trabajo de Carácter Psicosocial

\begin{tabular}{|c|c|c|c|c|c|c|c|c|c|c|}
\hline \multirow{2}{*}{ Factor } & \multicolumn{2}{|c|}{ Muy bueno } & \multicolumn{2}{|c|}{ Bueno } & \multicolumn{2}{|c|}{ Normal } & \multicolumn{2}{|c|}{ Regular } & \multicolumn{2}{|c|}{ Nocivo } \\
\hline & $f r$ & $\%$ & $f r$ & $\%$ & $f r$ & $\%$ & $f r$ & $\%$ & $f r$ & $\%$ \\
\hline Cultura de la Organización y gestión & 10 & 5.0 & 81 & 40.7 & 19 & 9.5 & 78 & 39.2 & 11 & 5.5 \\
\hline Papel o rol en la organización & 30 & 15.1 & 107 & 53.8 & 12 & 6.0 & 49 & 24.6 & 1 & 0.5 \\
\hline $\begin{array}{l}\text { Interrelación trabajo con problemas } \\
\text { familiares o sociales }\end{array}$ & 29 & 14.6 & 103 & 51.8 & 33 & 16.6 & 30 & 15.1 & 4 & 2.0 \\
\hline Relaciones interpersonales & 45 & 22.6 & 86 & 43.2 & 22 & 11.1 & 43 & 21.6 & 3 & 1.5 \\
\hline Carga y ritmo de trabajo & 23 & 11.6 & 103 & 51.8 & 18 & 9.0 & 53 & 26.6 & 2 & 1.0 \\
\hline Ambientes laborales & 10 & 5.0 & 58 & 29.1 & 28 & 14.1 & 92 & 46.2 & 11 & 5.5 \\
\hline Equipos y agentes físicos & 34 & 17.1 & 81 & 40.7 & 34 & 17.1 & 45 & 22.6 & 5 & 2.5 \\
\hline $\begin{array}{l}\text { Concepción de las tareas del puesto de } \\
\text { trabajo }\end{array}$ & 20 & 10.1 & 53 & 26.6 & 54 & 27.1 & 58 & 29.1 & 14 & 7.1 \\
\hline Amortiguadores del riesgo psíquico & 32 & 16.1 & 94 & 47.2 & 11 & 5.5 & 54 & 27.1 & 8 & 4.0 \\
\hline Características de la organización & 51 & 25.6 & 104 & 52.3 & 9 & 4.5 & 34 & 17.1 & 1 & 0.5 \\
\hline Características personales & 62 & 31.2 & 89 & 44.7 & 19 & 9.5 & 29 & 14.6 & 0 & 0.0 \\
\hline Respuesta cognitiva-emocional & 50 & 25.1 & 85 & 42.7 & 12 & 6.0 & 46 & 23.1 & 6 & 3.0 \\
\hline Respuesta conductual & 62 & 31.2 & 99 & 49.7 & 13 & 6.5 & 23 & 11.6 & 2 & 1.0 \\
\hline Respuesta fisiológica & 62 & 31.2 & 89 & 44.7 & 7 & 3.5 & 40 & 20.1 & 1 & 0.5 \\
\hline
\end{tabular}

6 Nota: Elaboración propia a partir de la batería Condiciones de Trabajo de Carácter Psicosocial (Carrión et al., 2014b). 
Correlación de Pearson entre las dimensiones psicosociales de la Batería Condiciones de Trabajo de Carácter Psicosocial y el Desgaste Psíquico

\begin{tabular}{|c|c|c|c|c|}
\hline \multirow{2}{*}{ Dimensiones Psicosociales } & Contexto de Trabajo & Contenido de Trabajo & Factores Individuales & Desgaste Psíquico \\
\hline & $r$ & $r$ & $r$ & $r$ \\
\hline Contexto de Trabajo & & $0.40^{*}$ & $0.61^{*}$ & $0.48^{*}$ \\
\hline Contenido de Trabajo & & & $0.37^{*}$ & $0.65^{*}$ \\
\hline Factores Individuales & & & & $0.51^{*}$ \\
\hline
\end{tabular}

Nota: Elaboración propia a partir de la batería Condiciones de Trabajo de Carácter Psicosocial (Carrión et al., $2014 b)$ * $<$.01.

\section{Líneas de acción para el control de las condiciones de trabajo psicosociales}

Elaborar o complementar una política de seguridad y salud en el trabajo que contemple los factores de riesgo psicosocial; la NOM-035-STPS-2018, propone un ejemplo de política de prevención de riesgos psicosociales en el que la organización asume un compromiso para un mejor entorno laboral, atendiendo los factores más desfavorables con el propósito de lograr un trabajo digno.

Implementar medidas encaminadas a la prevención de los factores de riesgo psicosocial y de la violencia laboral, así como la promoción de un entorno organizacional favorable a partir de la mejora en los factores del contenido y del contexto de trabajo. Así mismo implementar estrategias complementarias para conservar y/o mejorar los factores individuales y la percepción del desgaste psíquico.

La organización, para promover el sentido de pertenencia de los trabajadores a la misma, hace hincapié en que las personas son importantes para el centro de trabajo, ya que éste no puede funcionar si no se cuenta con la colaboración de todo el personal

Se propone que el talento humano de todos los niveles de la organización participe del proceso de mejora, desde el diagnóstico hasta la ejecución de los planes de acción. Lo cual permite un mayor sentido de pertenencia y la apropiación de las medidas consensuadas lo que optimiza la gestión integral.

Realizar programas de identificación de las características del puesto de trabajo, así como de los posibles riesgos y de cómo actuar en caso de accidente (Moreno \& Báez, 2010). Proporcionar conocimientos y competencias con desarrollo personal y profesional al contenido de trabajo que se debe desempeñar para que sea valioso y de utilidad en la organización (Patlán, 2016; Posada, 2013).

Los trabajadores que sometidos a periodos de estrés prolongados puedan desarrollar problemas graves de salud física, como enfermedades cardiovasculares o problemas musculo-esqueléticos, deberán ser derivados para su atención por un especialista que haga la evaluación específica y prescriba el tratamiento adecuado, para evitarles efectos negativos a ellos y a la empresa, los cuales se traducen en un aumento del ausentismo, presentismo y en el incremento de índices de accidentes y lesiones laborales.

En esta industria, para lograr la definición precisa de responsabilidades para los trabajadores, se requiere revisar la organización del trabajo y la estructura organizacional para enriquecer los puestos de trabajo, diseñar el programa de capacitación del personal y posteriormente actualizar los manuales de organización, difundirlos y darles seguimiento.

\section{Discusión}

Los resultados mostraron la necesidad de prestar atención a acciones y medidas de intervención para propiciar un buen entorno laboral y disminuir la percepción desfavorable de los factores psicosociales. En 
concordancia con el objetivo planteado en este estudio, se demostró que los trabajadores no perciben como suficientes las medidas que ha tomado la organización hasta el momento de acuerdo con los resultados, situación que presenta áreas de oportunidad de estudio y de acción en la cultura organizacional, el liderazgo, la calidad de vida laboral, por ende, la motivación por el trabajo, el compromiso laboral y la disposición al esfuerzo (Posada, 2013), pueden desencadenar una serie de situaciones encaminadas a la rotación, al ausentismo, quejas por parte del personal e incluso síntomas asociados afectando la salud ocupacional (Luceño et al., 2017; Marín \& Piñeros, 2018; Vera et al., 2015).

En esta investigación la percepción de los factores psicosociales fue más desfavorable en dos de las cuatro dimensiones que evalúa la Batería CTCPS-MAC en Contexto de Trabajo, particularmente en Cultura de la Organización y Gestión, y Contenido de Trabajo. Estos resultados se apartaron de lo encontrado en el estudio de Carrión et al. (2014b) en Colombia, ya que dichas dimensiones fueron las más favorables (considerando los niveles muy bueno y bueno) en un $85.2 \%$ y $78.1 \%$ respectivamente, mientras que las más desfavorables fueron Factores Individuales y Desgaste Psíquico con el menor porcentaje en los niveles normal, regular y nocivo.

En la investigación de Saldaña et al. (2014) en el sur de Jalisco, México, se mostraron porcentajes favorables (muy bueno y bueno) en Contexto de Trabajo con un 83.0\%, Factores Individuales con 90.5\% y Desgaste Psíquico con un 84.8\%, mientras que en Contenido de Trabajo fue de sólo un $52.0 \%$. Asimismo, en el estudio de Anaya (2016) en una organización pública de educación superior del occidente de México, la percepción de cada una de las dimensiones resultó favorable (niveles en muy bueno y bueno) siendo para Contexto de Trabajo un 88.0\%, para Contenido de Trabajo fue un 84.0\%, en Factores Individuales con 98.0\% y Desgaste Psíquico con 93.0\%. A pesar de las diferencias, existe un punto en común entre estos estudios, en la dimensión Contenido de Trabajo hubo el porcentaje más bajo para estos casos, indicando que sí existe una percepción desfavorable en comparación al resto de las dimensiones.

El personal evaluado y su percepción acerca de sus Factores Individuales y Desgaste Psíquico, resultó favorable por una proporción cercana al $85 \%$ de los trabajadores, mientras que la percepción del Contexto de trabajo ronda $40 \%$ y del Contenido de trabajo el 55\%. Esto podría deberse a que dicha percepción desfavorable surge de la influencia que tienen los factores psicosociales de los problemas inherentes de las personas, las condiciones de trabajo y de su organización, generando la posibilidad de caer en el Desgaste Psíquico (Cacivio, 2017; Hernán, 2018), es por ello que proponer acciones de mejora para aquellas condiciones relacionadas con la organización que impactan en los factores psicosociales, contribuye a promover tanto un ambiente de trabajo óptimo para cada integrante de la organización, como a mantener la salud física y mental de los trabajadores (Hernán, 2018; Lima, Moro, \& Cotrim, 2018; Potter et al., 2019).

\section{Conclusión}

El objetivo de evaluar la percepción que tiene el personal de una industria manufacturera sobre los factores de riesgo psicosocial se cumplió, debido a que se logró su descripción y mostrar su relación con el desgaste psíquico. Realizar investigaciones en las organizaciones, es encontrar un entorno dinámico, por lo que es necesario analizar cualquier información obtenida para lograr el mayor provecho posible con el objetivo de brindar datos precisos y estrategias de cambio para la mejora de dicho entorno laboral.

Se concluye que para tener una evaluación más afinada y poder crear líneas de intervención más precisas en los factores psicosociales percibidos por el personal, se requiere realizar otros estudios específicos en las dimensiones o factores más desfavorables, que involucren a expertos y un equipo multidisciplinario para corroborar que las condiciones de trabajo a las que los trabajadores están expuestos, sean las óptimas

8 para laborar y así cumplir con los requerimientos que solicita la Normativa, en este caso la mexicana. Cabe 
mencionar, que, durante el estudio, las limitantes encontradas están referidas a que el instrumento es multidimensional por lo que se requieren evaluaciones específicas, así como complementar la información mediante estrategias cualitativas. Por otra parte, se rescata que la información obtenida de los participantes durante la recolección de datos, fue valiosa para aclarar las dudas y cumplir objetivos tanto de los participantes como de los investigadores.

Finalmente, se plantea la necesidad de seguir indagando en los factores psicosociales y sus medidas de control para un mejor entorno laboral, ya que si bien se encuentran evidencias de consultorías e intervenciones, éstas no se describen en su totalidad en el ámbito científico, lo cual dificulta su mejora y efectividad en las organizaciones, por ello, se necesita un mayor aporte para cubrir este vacío científico.

\section{Contribución}

E.A. LUNA-CHÁVEZ desarrolló el concepto y el diseño de la investigación. A. ANAYA-VELASCO trabajó sobre el análisis e interpretación de los datos. E. RAMÍREZ-LIRA contribuyó en la revisión y la versión final del artículo.

\section{Referencias}

Agencia Europea para la Salud y la Seguridad en el Trabajo. (2016). Los riesgos psicosociales y el estrés en el trabajo, nota descriptiva. Recuperado el abril 3, 2018 de https://osha.europa.eu/es/themes/psychosocial-risks-and-stress

Anaya, A. (2016). Bienestar Integral CUSur: iSalud y seguridad para todos! Informe de resultados. Ciudad Guzmán: Universidad de Guadalajara.

Anaya, A., Saldaña, C., \& Ramírez, E. (2017). Acciones de intervención organizacional para mejorar las condiciones de trabajo en el Departamento de Obras Públicas de un ayuntamiento del Sur de Jalisco (México). Revista Científica Salud Uninorte, 33(3), 373-382.

Cacivio, R. (2017). Hablemos de nuestro trabajo: riesgos psicosociales en entornos de trabajo de extensionistas agropecuarios de la Argentina. Pesquisa Empírica, 13(1), 39-55.

Carrión, M. (2014a). Batería para el estudio de las condiciones de trabajo de carácter psicosocial. España: Editorial Académica Española.

Carrión, M., Gutiérrez, A., \& López, F. (2014b). Condiciones de trabajo psicosociales y desgaste psíquico en trabajadores de diversos sectores empresariales de Centro-Oriente, Suroccidente y región Caribe en Colombia. Revista Científica Salud Uninorte, 30(3), 311-322.

Comité Mixto OIT/OMS de Medicina del Trabajo. (1984). Factores psicosociales en el trabajo: naturaleza, incidencia y prevención. Ginebra: Oficina Internacional del Trabajo. Recuperado el junio 15, 2018, de http://www. factorespsicosociales.com/wp-content/uploads/2019/02/FPS-OIT-OMS.pdf

Coolican, H. (2005). Métodos de investigación y estadística en psicología (3a ed.). México: Manual Moderno.

García, J., Puebla, M., Herrera, P., \& Daudén, E. (2016). Desmotivación del personal sanitario y síndrome de burnout. Control de las situaciones de tensión. La importancia del trabajo en equipo. Actas Dermo-Sifiliográficas, 107(5), 400-406.

Hernán, C. (2018). Incidence of the psychosocial risk at work: A case study of technical teachers at city of Morón. Advances in Intelligent Systems and Computing, 826, 768-777.

Instituto Nacional de Seguridad e Higiene en el Trabajo. (1995). Factores psicosociales: metodología de evaluación. Recuperado el abril 3, 2018, de http://www.insht.es/InshtWeb/Contenidos/Documentacion/FichasTecnicas/NTP/ Ficheros/401a500/ntp_443.pdf

Instituto Sindical del Trabajo, Ambiente y Salud. (2015). Riesgos psicosociales, nota descriptiva. Recuperado el abril 3, 2018, de http://istas.net/salud-laboral/peligros-y-riesgos-laborales/riesgos-psicosociales

Katenkamp, O., Dechman, U., Georg, A., Meyn, C., Peter, A., \& Peter, G. (2014). Change-Management und seine psychosozialen Belastungen- eine interdisziplinäre Betrachtung über die Autonomie der Arbeit. Abhandlungen, 23, 77-91.

Kerlinger, F., \& Lee, H. (2002). Investigación del comportamiento: métodos de investigación en ciencias sociales. México: McGraw Hill. 
Labarthe, J., \& Fillat, L. (2017). Conciliación familia-trabajo y conciliación trabajo-familia en una institución financiera. In L. Magalhaes \& A. Barciela (Orgs.), Saúde Psíquica e Trabalho. Campo Grande: UCDB.

Lima, I., Moro, A., \& Cotrim, P. (2018). Capacity index for work, psychosocial risk of work and musculoskeletal symptomatology in workers of a meat processing industry in Portugal. Advances in Intelligent Systems and Computing, 820, 289-295.

Luceño, L., Talavera, B., Martín, J., \& Escorial, S. (2017). Factores de riesgo psicosocial como predictores del bienestar laboral: un análisis SEM. Ansiedad y Estrés, 23(2-3), 66-70.

Marín, C., \& Piñeros, O. (2018). Organizational management, human resources and mobbing: Findings in Colombia. Advances in Intelligent Systems and Computing, 791, 110-115.

Mathisen, G., Bronnick, K., Arntzen, K., \& Vestly, L. (2016). Identifying and managing psychosocial risks during organizational restructuring: It's what you do and how you do it. Safety Science, 100, 20-29.

Mendoza, M. (2013). Costo e impacto de los riesgos de trabajo (Tesis de Maestría no-publicado). Ciudad de México.

Moreno, B., \& Báez, C. (2010). Factores y riesgos psicosociales, formas, consecuencias, medidas y buenas prácticas. España: Instituto Nacional de Seguridad e Higiene en el Trabajo.

Organización Internacional del Trabajo. (2013). Día mundial de la seguridad y salud em el trabajo. Turín: Centro Internacional de Formación de la OIT.

Patlán, J. (2016). Calidad de vida en el trabajo. Ciudad de México: Manual Moderno.

Posada, A. S. (2013). Sentido de pertenencia. In F. T. Álvarez \& A. S. Posada, Gestión del clima organizacional: intervención basada en evidencias. Medellín: Centro de Investigación en Comportamiento Organizacional.

Potter, R., O'Keeffe, V., Leka, S., Webber, M., \& Dollard, M. (2019). Analytical review of the australian policy context for work-related psychological health and psychosocial risks. Safety Science, 111, 37-48.

Saldaña, C., Ramírez, E., Anaya, A., \& Baeza, M. (2014). Evaluación de los riesgos psicosociales con el instrumento CTCPS-MAC-batería para el estudio de las condiciones de trabajo de carácter psicosocial- en el Departamento de Obras Públicas de un ayuntamiento del sur de Jalisco (México). Revista Científica Salud Uninorte, 30(3), 293-301.

Secretaría del Trabajo y Previsión Social (México). (2014). Reglamento Federal de Seguridad y Salud en el Trabajo. Diario Oficial de la Federación. Recuperado el mayo 8, 2018 de http://www.dof.gob.mx/nota_detalle. php?codigo $=5368114 \&$ fecha $=13 / 11 / 2014$

Secretaría del Trabajo y Previsión Social (México). (2015). Marco Normativo. Recuperado el abril 3, 2018, de http://www. stps.gob.mx/bp/secciones/dgsst/normatividad.html

Secretaría del Trabajo y Previsión Social (México). (2018). Norma Oficial Mexicana NOM-035-STPS-2018. Factores de riesgo psicosocial Identificación, análisis y prevención. Diario Oficial de la Federación. Recuperado el octubre 30, 2018 de https://www.dof.gob.mx/nota_detalle.php?codigo=5541828\&fecha=23/10/2018

Thomascheck, A., Lütke, S., Melzer, M., Debitz, U., \& Buruck, G. (2017). Measuring work-related psychosocial and physical risk factors using workplace observations: A validation study of the "Healthy Workplace Screening". Safety Science, 101, 197-208.

Unda, S., Uribe, F., Jurado, S., García, M., Tovalín, H., \& Júarez, A. (2016). Elaboración de una escala para valorar los factores de riesgo psicosocial en el trabajo de profesores universitarios. Journal of Work and Organizational Psychology, $32,67-74$.

Vazquez, A., Pianezolla, M., \& Hutz, C. (2018). Assesment of psychosocial factors at work: A systematic review. Estudos de Psicologia (Campinas), 35(1), 5-13.

Vera, A., Carrasco, A., Da Costa, S., \& Páez, D. (2015). Factores psicosociales del presentismo en trabajadores del Sistema de Salud chileno. Revista de Psicología del Trabajo y de las Organizaciones, 31(2), 119-128.

Vestly, L., Leka, S., \& Zwetsloot, G. (2017). Tailoring psychosocial risk assessment in the oil and gas industry by exploring specific and common psychosocial risks. Safety and Health at Work, 9, 63-70.

Weissbrodt, R., Arial, M., Graf, M., Iff, S., \& Giauque, D. (2018). Preventing psychosocial risks at work: An evaluation study of labour inspectorate interventions. Safety Science, 110, 355-362.

Recibido: Noviembre 27, 2018

Versión final: Mayo 8, 2019

10

Aprobado: Agosto 22, 2019 